\title{
Knockdown of HIP1 expression promotes ligand-induced endocytosis of EGFR in HeLa cells
}

\author{
DAN LI $^{1 *}$, FENGLIN CHEN $^{1 *}$, JIAN DING $^{2}$, NA LIN $^{1}$, ZONGHAI LI $^{3}$ and XIAOZHONG WANG ${ }^{1}$ \\ ${ }^{1}$ Department of Gastroenterology, Union Hospital of Fujian Medical University, Gulou, Fuzhou, Fujian 350001; \\ ${ }^{2}$ Department of Gastroenterology, The First Affiliated Hospital of Fujian Medical University, Taijiang, Fuzhou, \\ Fujian 350005; ${ }^{3}$ State Key Laboratory of Oncogenes and Related Genes, Shanghai Cancer Institute, \\ Shanghai Jiaotong University School of Medicine, Shanghai 200032, P.R. China
}

Received April 20, 2017; Accepted September 25, 2017

DOI: $10.3892 /$ or.2017.6025

\begin{abstract}
Huntington-interacting protein 1 (HIP1) is associated with various tumor types; however, its precise functions in tumor cells are unclear. In this study, the effects of HIP1 on the degradation of EGFR, which have important roles in carcinogenesis after EGF stimulation, were examined. After screening 17 cell lines, the coexpression of HIP1 and EGFR was detected in HeLa cells. Accordingly, the expression of HIP1 was knocked down in HeLa cells using various HIP1 siRNA sequences. The endocytosis of EGFR and localization of clathrin in HeLa cells were examined after stimulation by EGF at various concentrations (i.e., 1.5 and $100 \mathrm{ng} / \mathrm{ml}$ ). After HIP1 expression was blocked by siRNAs, EGFR endocytosis was accelerated and this effect was dependent on the EGF concentration. This endocytosis was colocalized with clathrin expression. These findings indicate that the inhibition of HIP1 can accelerate the endocytosis and degradation of EGFR. Furthermore, they suggest that HIP1 is a potential therapeutic target for various cancer types, particularly those with high EGFR expression, but further research is needed to examine this hypothesis.
\end{abstract}

\section{Introduction}

Huntington-interacting protein 1 (HIP1) was originally characterized using the yeast two-hybrid system in 1997 (1). HIP1

Correspondence to: Professor Xiaozhong Wang, Department of Gastroenterology, Union Hospital of Fujian Medical University, 29 Xinquan Road, Gulou, Fuzhou, Fujian 350001, P.R. China

E-mail: drwangxiaozhong@sohu.com

${ }^{*}$ Contributed equally

Abbreviations: HIP1, Huntington-interacting protein 1; AP2, adaptor protein 2; GnT-Va, $N$-acetylglucosaminyltransferase $\mathrm{Va}$

Key words: EGFR, endocytosis, Huntington-interacting protein 1, siRNA, tumor cells and its only known mammalian homolog HIP1-related (HIP1r) are involved in neurodegeneration based on the finding that HIP1 interacts with the Huntington protein, which is mutated in Huntington's disease, and acts as a component of the endocytic machinery, binding to clathrin, AP2 and actin (2-4).

The overexpression of HIP1 is correlated with brain (5), colon (6), and breast cancers (7). Additionally, HIP1 overexpression in glioblastoma and oligodendroglioma results in a prolonged half-life of growth factor receptors, such as EGFR and PDGF- $\beta$ R (5). EGFR and its downstream pathways are important for tumor cell invasion and proliferation. The degradation of EGFR is partially achieved by the internalization of activated EGFR and its degradation in lysosomes $(8,9)$.

The stabilization of EGFR levels by HIP1 in cancer cells suggests that HIP1 is related to endocytosis and EGFR degradation, but this relationship has not been definitively established. In this study, the effect of HIP1 on the first step of EGFR endocytosis and the mechanisms by which HIP1 mediates cancer cell proliferation in an EGFR-dependent manner were examined.

\section{Materials and methods}

Materials. EGF and Alexa Fluor 647-EGF were purchased from Sigma-Aldrich (St. Louis, MO, USA). Human PKR siRNA (SR303767) and control siRNA (SR30004) were purchased from OriGene (Rockville, MD, USA). The siRNAs against HIPl were purchased from Ambion (Foster City, CA, USA). Antibodies against clathrin and EGFR were purchased from Abcam (Cambridge, UK). Tetramethylrhodamine isothiocyanate-labeled affinity purified goat anti-mouse IgG and goat anti-rabbit $\operatorname{IgG}(\mathrm{H}+\mathrm{L})$ were purchased from KPL (Guildford, UK). Electrochemiluminescence kit was bought for Boshide (Wuhan, China). All other chemicals were purchased from Sigma-Aldrich.

Cell culture. Human hepatocarcinoma cells lines 7402, 7703, 7721, Hep3B, HepG2, H460, SPCA1, SKOV-3, HeLa, and MCF-7 and glioma cells U87, U251, C33a, PC-3, NCI-H1299, NCI-H446, and K562 were cultured in Dulbecco's modified Eagle's medium (DMEM) supplemented with $10 \%$ fetal bovine serum, $2 \mathrm{mM}$ L-glutamine, and $1 \mathrm{X}$ antibiotic-antimycotic 
solution (15240-096; Invitrogen, Carlsbad, CA, USA) at $37^{\circ} \mathrm{C}$ in a humidified atmosphere with $5 \% \mathrm{CO}_{2}$.

siRNA and transfection. The sequences of siRNAs targeting human $H I P 1$ were as follows: siRNA1, taattgagcgactatacagag; siRNA2, acagcgatatagcaagctaaa; siRNA3, accgcttcatggag cagttta. These siRNAs were designed using siRNA Target Finder developed by Ambion, Inc. Cells were transfected with siRNA using Lipofectamine 2000 (11668-019; Invitrogen) according to the manufacturer's protocol. Transfection was confirmed by an immunoblot analysis.

Western blot assay. Cells were collected, pelleted, and lysed in ice-cold lysis buffer $(25 \mathrm{mmol} / \mathrm{l}$ Tris- $\mathrm{HCl}, 1 \mathrm{mmol} / \mathrm{l}$ edetic acid, $150 \mathrm{mmol} / 1 \mathrm{NaCl}, 50 \mathrm{mmol} / \mathrm{l} \mathrm{NaF}, 1 \%$ Triton-100, $1 \mathrm{mmol} / \mathrm{l} \mathrm{PMSF}, 1 \mathrm{mg} / 1$ leupeptin, $1 \mu \mathrm{mol} / 1$ aprotinin, $\mathrm{pH}$ 7.6). Cell lysates were centrifuged at $4^{\circ} \mathrm{C}$ and $12,000 \mathrm{~g} / \mathrm{min}$, rotated with polyclonal rabbit anti-human antibody for $2 \mathrm{~h}$, followed by precipitation with Protein A Sepharose at $4^{\circ} \mathrm{C}$ overnight. Beads were washed five times with cold wash buffer $(20 \mathrm{mmol} / \mathrm{l}$ Tris, pH 7.8, $150 \mathrm{mmol} / 1 \mathrm{NaCl}, 1 \mathrm{mmol} / \mathrm{l}$ EDTA, $0.1 \%$ Triton X-100, $100 \mu \mathrm{M}$ PMSF and $1 \mathrm{mmol} / 1 \mathrm{Na}_{3} \mathrm{VO}_{4}$ ) and the bound protein was eluted with Laemmli sample buffer and separated by sodium dodecyl sulfate polyacrylamide gel electrophoresis (SDS-PAGE). After SDS-PAGE, the protein was transferred to a nitrocellulose membrane using a semidry transfer apparatus (Bio-Rad, Hercules, CA, USA), blocked with $3 \%$ bovine serum albumin in TBST [ $10 \mathrm{mmol} / 1$ Tris ( $\mathrm{pH} 8.0), 150 \mathrm{mmol} / 1$ $\mathrm{NaCl}, 0.1 \%$ Tween-20], incubated at room temperature for $1 \mathrm{~h}$ with rabbit anti-human HIP1, EGFR, and GAPDH antibodies, rotated for $1 \mathrm{~h}$ at room temperature of $25^{\circ} \mathrm{C}$, and washed with TBST three times. The protein on the nitrocellulose membrane was then detected by electrochemiluminescence using a kit with a goat anti-rabbit antibody according to the manufacturer's instructions. Based on the western blotting results, HeLa cells exhibited high coexpression of HIP1 and EGFR and accordingly were used in subsequent experiments.

EGF-EGFR internalization assay. HeLa cells were plated in 6-well dishes and grown to $50 \%$ confluency. After cells were serum-starved for $2 \mathrm{~h}$ at $37^{\circ} \mathrm{C}$, they were incubated with serum-free DMEM containing Alexa Fluor 647-EGF at a final concentration of $1.5 \mathrm{ng} / \mathrm{ml}$ or $100 \mathrm{ng} / \mathrm{ml}$ for $1 \mathrm{~h}$ at $4^{\circ} \mathrm{C}$. Cells were then incubated at $37^{\circ} \mathrm{C}$ for $1 \mathrm{~h}$ to allow internalization. EGFR internalization was stopped by placing the cells at $4^{\circ} \mathrm{C}$. The cells were then washed three times for 10 min with PBS, fixed, and permeabilized with $4 \%$ paraformaldehyde and $0.1 \%$ Triton X-100 in PBS for 10 min. They were then washed with PBS again at room temperature and visualized under a Zeiss LSM confocal microscope (Oberkochen, Germany).

Colocalization assay. EGFR internalization experiments were carried out as described previously. The cells were blocked with 5\% normal goat serum in PBS for $30 \mathrm{~min}$, washed three times for 5 min each with PBS, and then incubated with mouse anti-clathrin monoclonal antibody (Abcam) diluted 1:50 in PBS for $3 \mathrm{~h}$ at room temperature. After another three washes for 10 min each with PBS, tetramethylrhodamine isothiocyanate-labeled affinity-purified goat anti-mouse $\operatorname{IgG}$ and goat anti-rabbit IgG (H+L; KPL) diluted 1:50 in PBS
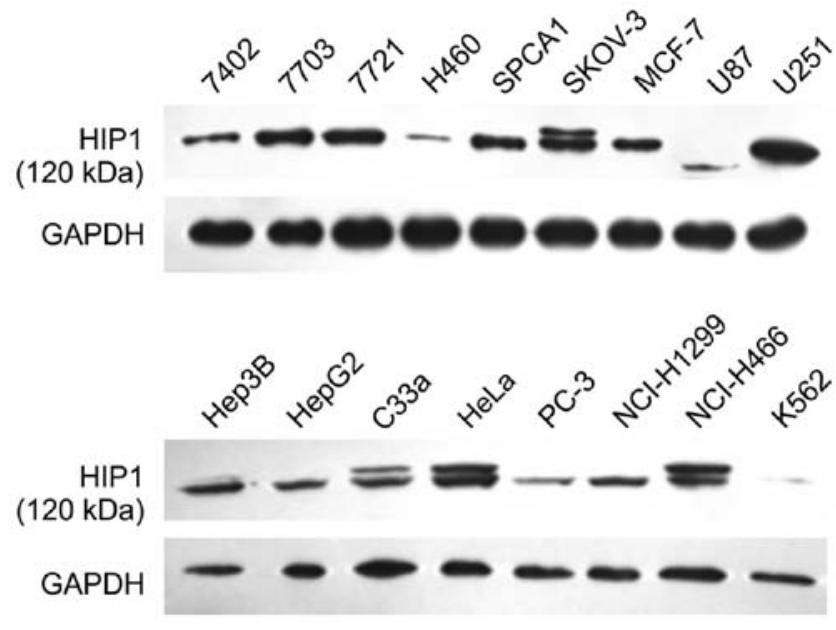

Figure 1. HIP1 expression in seventeen cell lines measured by western blotting. The cell lines were 7402, 7703, 7721, Hep3B, HepG2, H460, SPCA1, SKOV-3, HeLa, MCF-7, glioma cells U87, U251, C33a, PC-3, NCI-H1299, NCI-H446, and K562 cells. 7703, 7721, SKOV-3, U251, and HeLa cells exhibited higher expression levels of HIP1 than those of other cell lines.

were added and incubated for $30 \mathrm{~min}$. Following a final rinse (3x10 min) with PBS, the cells were visualized under a Zeiss LSM confocal microscope.

Image analysis. Images were collected using a Zeiss LSM510-Meta laser scanning confocal microscope with a 63x water immersion objective. Colocalization was calculated using Image J (NIH, Bethesda, MD, USA) with the JACoP plug-in to estimate Manders coefficients with automated thresholding. Statistical analyses were implemented in GraphPad Prism (GraphPad, La Jolla, CA, USA).

Statistical analysis. Based on the image analysis data, differences among treatment groups were examined by analysis of variance (ANOVA). Data are represented as means \pm SEM of three experiments. $\mathrm{P}<0.05$ was considered statistically significant. When significant differences were detected, specific post-hoc comparisons between treatment groups were performed using Student-Newman-Keuls tests.

\section{Results}

Expression of HIP1 and EGFR in tumor cell lines. Using 17 cell lines, western blotting was performed to identify cells with high expression levels of both HIP1 and EGFR. As shown in Fig. 1, the 7703, 7721, SKOV-3, U251, and HeLa cell lines exhibited higher expression levels of HIP1 than those of other cell lines. As shown in Fig. 2, the Hep3B, HeLa, PC-3, NCI-H1299, and NCI-H466 cell lines had higher expression levels of EGFR than those of other cell lines. Combining these results, the HeLa cell line had high expression levels of both HIP1 and EGFR.

Knockdown of HIPl in HeLa cells. Based on western blot assays, in HeLa cells, the expression of HIP1 was significantly blocked by siRNA1 (taattgagcgactatacagag) and siRNA2 (acagcgatatagcaagctaaa), both of which were more efficient compared with siRNA3 (accgcttcatggagcagttta) (Fig. 3). 


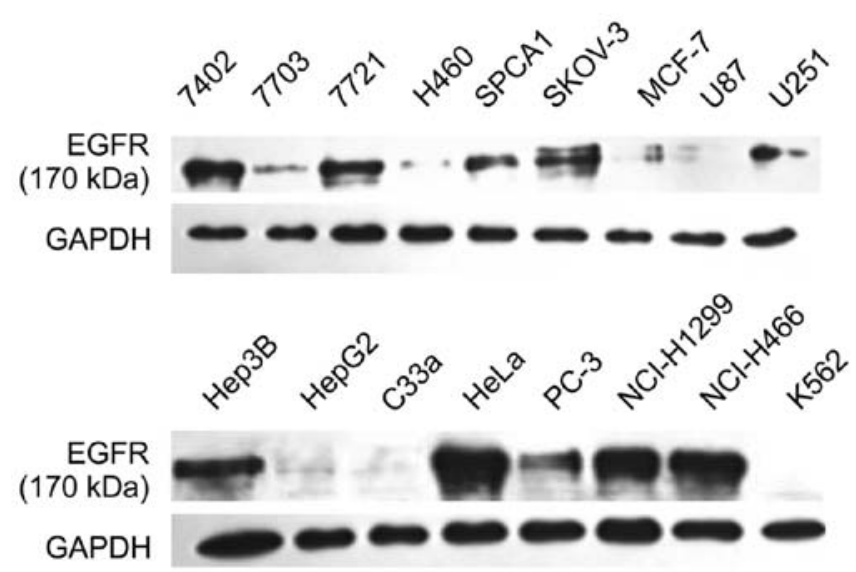

Figure 2. EGFR expression in seventeen cell lines measured by western blotting. The cell lines were 7402, 7703, 7721, Hep3B, HepG2, H460, SPCA1, SKOV-3, HeLa, MCF-7, glioma cells U87, U251, C33a, PC-3, NCI-H1299, NCI-H446, and K562 cells. Hep3B, HeLa, PC-3, NCI-H1299, and NCI-H466 cells exhibited higher expression levels of EGFR than those of other cell lines. Combined with the results presented in Fig. 1, HeLa cells had high expression of both HIP1 and EGFR.

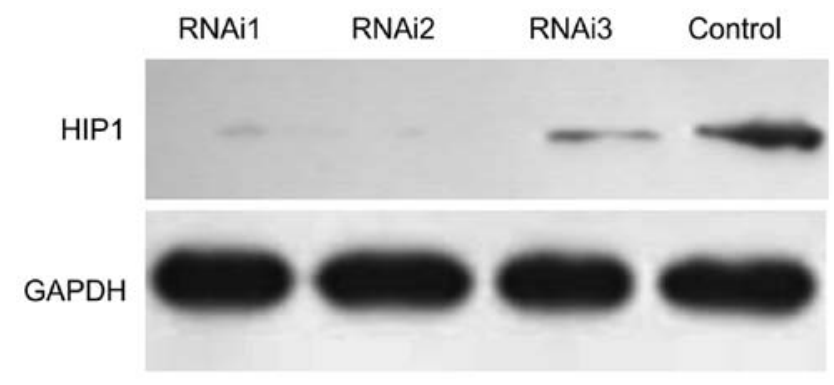

Figure 3. Expression levels of HIP1 in HeLa cells after HIP1 Stealth ${ }^{\mathrm{TM}}$ RNAi. Based on western blot assays, in HeLa cells, the expression of HIP1 was significantly blocked by siRNA1 (taattgagcgactatacagag) and siRNA2 (acagcgatatagcaagctaaa), both of which were more efficient than siRNA3 (accgcttcatggagcagttta).

Neither siRNA1 nor siRNA2 influenced the expression levels of EGFR and GAPDH in HeLa cells (Fig. 4).

NCI-H1299 cells exhibited high expression of EGFR and low expression of HIP1 (Figs. 1 and 2). Accordingly, NCI-H1299 was chosen as a control to examine the effects of HIPl siRNA. As shown in Fig. 5, the expression of HIP1 was significantly blocked by siRNA1, siRNA2 and siRNA3. The three siRNAs had no effect on the expression levels of EGFR and GAPDH in NCI-H1299 cells (Fig. 6).

Internalization of EGF-EGFR. After stimulation with $1.5 \mathrm{ng} / \mathrm{ml}$ EGF, endocytosis of EGF-bound EGFR was significantly accelerated after the expression of HIP1 was blocked (Fig. 7). After simulation with $100 \mathrm{ng} / \mathrm{ml} \mathrm{EGF}$, endocytosis of EGF-bound EGFR was also significantly accelerated after the expression of HIP1 was blocked (Fig. 8). There was no obvious difference between 1.5 and $100 \mathrm{ng} / \mathrm{ml}$ EGF with respect to EGFR endocytosis (Figs. 7 and 8).

Colocalization of EGF-EGFR and clathrin.Clathrin-mediated endocytosis was also accelerated after the expression of HIP1 was blocked, and exhibited a positive correlation with the

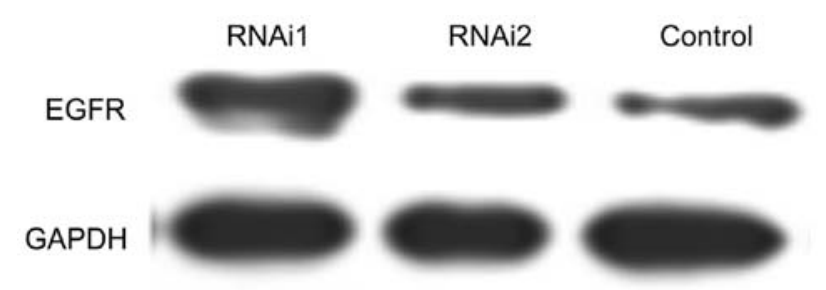

Figure 4. Expression levels of EGFR in HeLa cells with HIP1 Stealth RNAi. Neither siRNA1 nor siRNA2 had an effect on the expression levels of EGFR and GAPDH in HeLa cells.

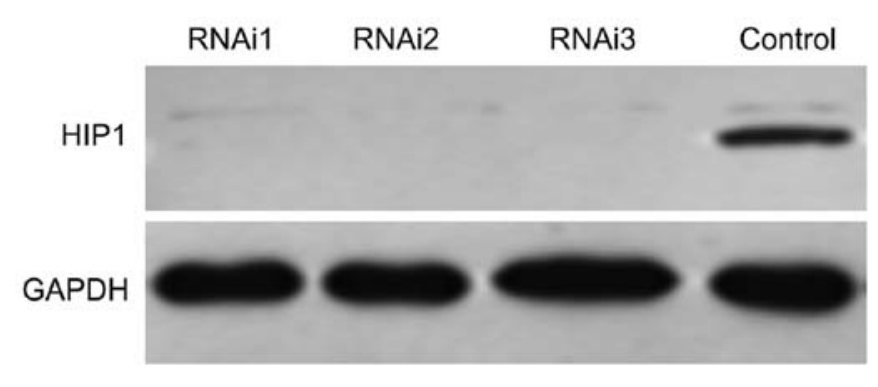

Figure 5. Expression levels of HIP1 in NCI-H1299 cells after HIP1 Stealth RNAi. Expression of HIP1 was significantly blocked by siRNA1, siRNA2 and siRNA3.

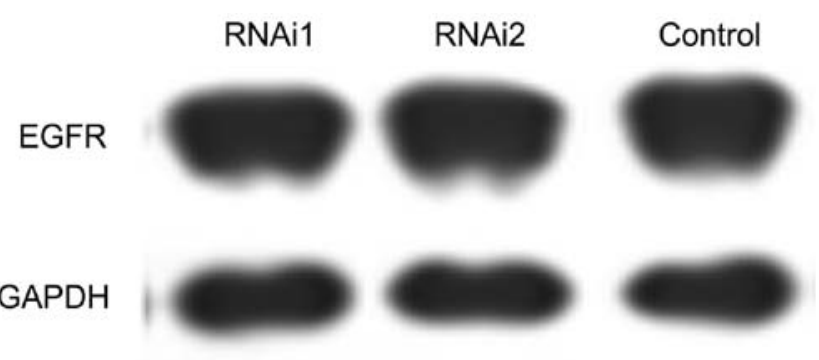

Figure 6. Expression levels of EGFR in NCI-H1299 cells after HIP1 Stealth RNAi. siRNAs had no effect on the expression of EGFR or GAPDH in NCI-H1299 cells.

internalization of EGF-EGFR for both $1.5 \mathrm{ng} / \mathrm{ml}$ (Fig. 7) and $100 \mathrm{ng} / \mathrm{ml}$ EGF (Fig. 8). There was no obvious difference between 1.5 and $100 \mathrm{ng} / \mathrm{ml}$ EGF with respect to clathrin endocytosis (Figs. 7 and 8). EGFR and clathrin were colocalized in the cytoplasm.

\section{Discussion}

Overactivation of EGFR signaling pathway is strongly associated with carcinogenesis, and it is becoming increasingly clear that impaired deactivation of EGFR may also be a mechanism in cancer. A major deactivation pathway for EGFR downregulation involves ligand-induced endocytosis of EGFR and subsequent degradation in lysosomes; this is important in carcinogenesis, e.g., in breast cancer (8). $\mathrm{N}$-acetylglucosaminyltransferase $\mathrm{Va}$ (GnT-Va) is involved in the EGF-induced downregulation of EGFR and intracellular signaling by inhibiting receptor endocytosis. When GnT-Va expression is knocked down in highly invasive human breast 

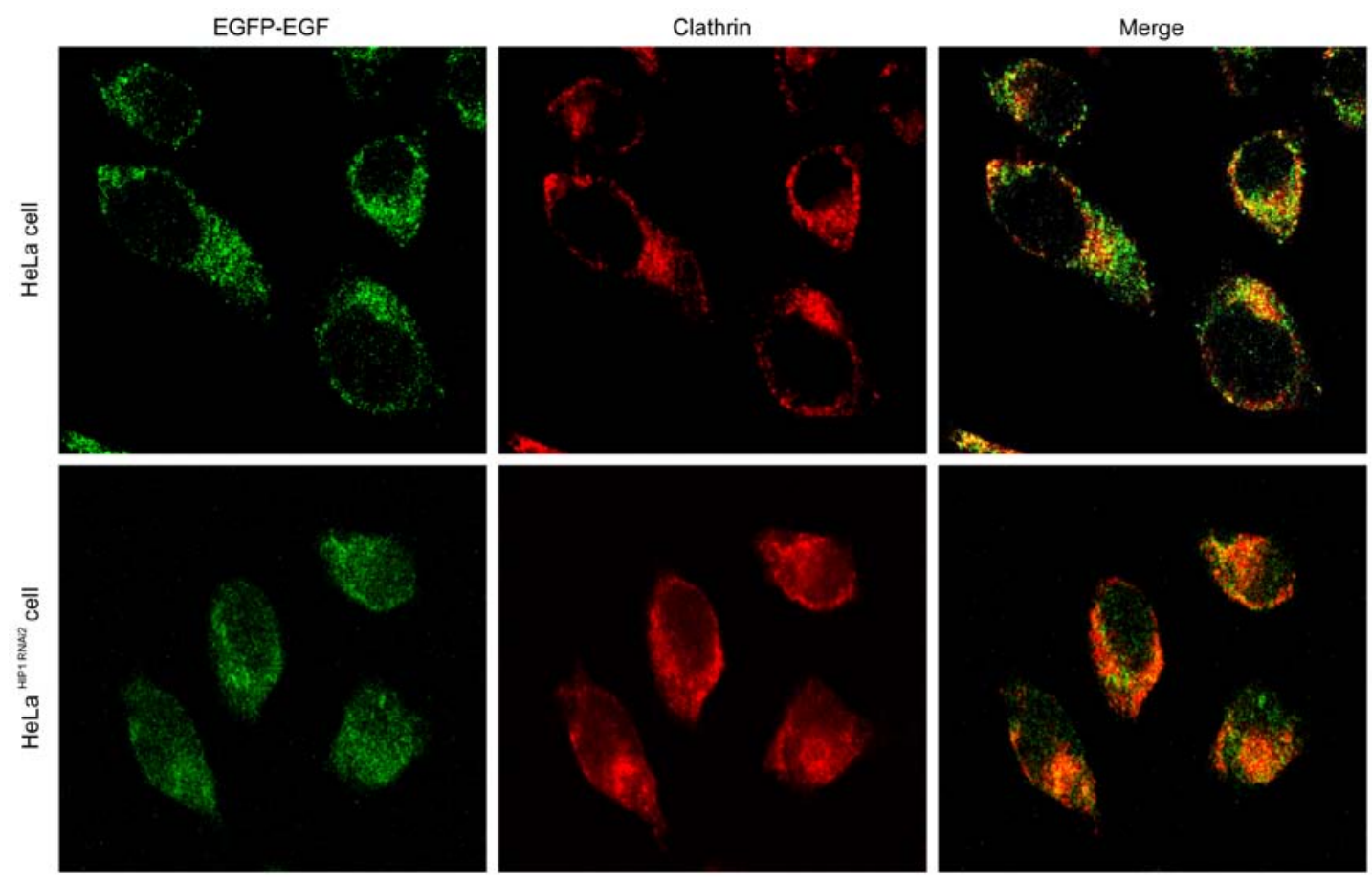

Figure 7. Endocytosis of EGFR and localization of clathrin in HeLa and HeLa HIP1 RNAi2 cells (1.5 ng/ml EGF stimulation). After $1.5 \mathrm{ng} / \mathrm{ml}$ EGF stimulation, endocytosis of EGF-bound EGFR and clathrin were accelerated after the expression of HIP1 was blocked. EGFR and clathrin were colocalized in the cytoplasm.
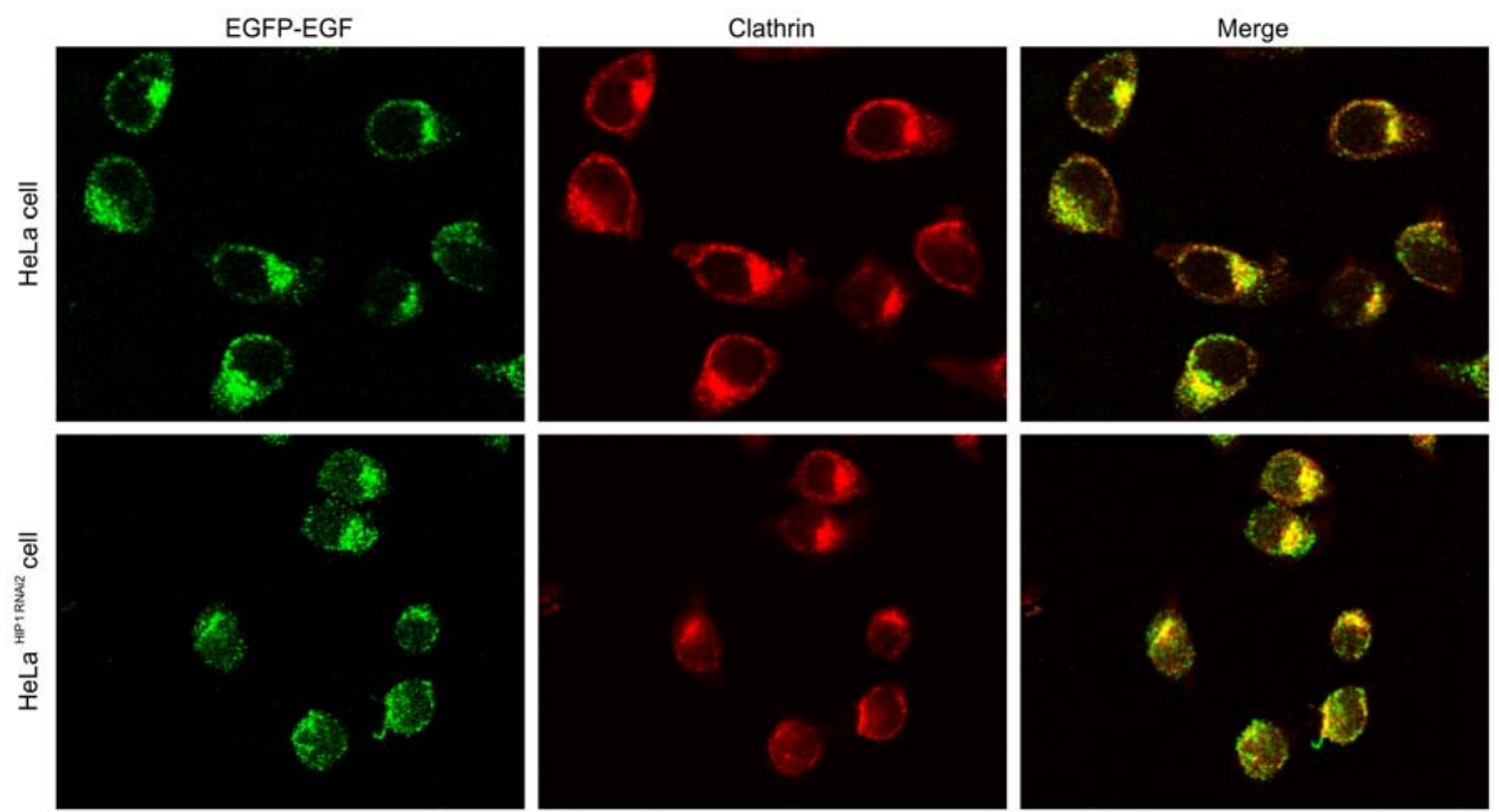

Figure 8. Endocytosis of EGFR and localization of clathrin in HeLa and HeLa HIP1 RNAi2 cells (100 ng/ml EGF stimulation). After $100 \mathrm{ng} / \mathrm{ml}$ EGF stimulation, endocytosis of EGF-bound EGFR and clathrin were accelerated significantly after the expression of HIP1 was blocked. EGFR and clathrin were colocalized in the cytoplasm.

cancer cells, ligand-induced downregulation of EGFR expression is inhibited via decreased EGFR endocytosis, resulting in delayed downstream signal transduction and inhibition of EGF-induced invasiveness phenotypes $(10,11)$.

HIP1 may be involved in the endocytosis of EGFR. Previous studies have demonstrated the colocalization of HIP1 and markers of clathrin-mediated endocytosis in neuronal cells and the enrichment of HIP1 on clathrin-coated vesicles purified from brain homogenates (5). HIP1 binds to clathrin adaptor protein 2 (AP2) and the terminal domain of the clathrin heavy chain, predominantly via a small fragment at amino acids 276-335. This region, a clathrin-box, contains consensus clathrin- and AP2-binding sites with high binding affinity to the terminal domain of the clathrin heavy chain and the ear domain of the AP2 subunit, respectively, leading to efficient stimulation of the clathrin assembly via its central 
helical domain by binding directly to the clathrin light chain (3-5). These results suggest that HIP1 has functional roles in clathrin-mediated endocytosis.

In this experiment, we screened 17 tumor cell lines to identify cells with high expression of both HIP1 and EGFR. HeLa cells had obviously high expression of both HIP1 and EGFR. Various siRNAs were designed to block the expression of HIP1 in HeLa cells to evaluate its inhibitory effects on EGFR endocytosis. Two siRNA sequences (taattgagcgactata cagag and acagcgatatagcaagctaaa, efficiently blocked HIP1 expression. We confirmed these results using NCI-H1299 cells as controls. After the blockage of HIP1 expression and stimulation with $1.5 \mathrm{ng} / \mathrm{ml} \mathrm{EGF,} \mathrm{EGFR} \mathrm{endocytosis} \mathrm{was} \mathrm{significantly}$ accelerated. The same results were obtained after stimulation with $100 \mathrm{ng} / \mathrm{ml}$ EGF. The acceleration of EGFR endocytosis was only correlated with HIP1 blockage. HIP1 can stabilize EGFR on cell surfaces by decreasing EGFR endocytosis. This process was correlated with clathrin endocytosis.

In the present study, we explored the role of HIP1 in the degradation of EGFR in cancer cells. This is the first analysis of EGFR and HIP1 coexpression in a large number of cell lines, and our results clearly demonstrated the effects of HIP1 inhibition on EGFR endocytosis. These findings may explain the proliferative and anti-apoptotic effects of HIP1 on tumor cells. HIP1 inhibition can accelerate EGFR endocytosis and degradation. These results also suggest a new method to treat carcinoma with high EGFR expression by targeting HIP1, but additional studies are needed to evaluate the clinical potential.

\section{Acknowledgements}

This study was supported by grants from the National Natural Science Foundation of China (81300321), the Key Discipline Foundation of Fujian Province (2012-149), and the Young and Middle-Aged Personnel Training Project of Fujian Province Health Department (2014-ZQN-ZD-9).

\section{References}

1. Kalchman MA, Koide HB, McCutcheon K, Graham RK, Nichol K, Nishiyama K, Kazemi-Esfarjani P, Lynn FC, Wellington C, Metzler M, et al: HIP1, a human homologue of $S$. cerevisiae Sla2p, interacts with membrane-associated huntingtin in the brain. Nat Genet 16: 44-53, 1997.

2. Legendre-Guillemin V, Metzler M, Charbonneau M, Gan L, Chopra V, Philie J, Hayden MR and McPherson PS: HIP1 and HIP12 display differential binding to F-actin, AP2, and clathrin. Identification of a novel interaction with clathrin light chain. J Biol Chem 277: 19897-19904, 2002.

3. Metzler M, Legendre-Guillemin V, Gan L, Chopra V, Kwok A, McPherson PS and Hayden MR: HIP1 functions in clathrinmediated endocytosis through binding to clathrin and adaptor protein 2. J Biol Chem 276: 39271-39276, 2001.

4. Mousavi SA, Malerød L, Berg T and Kjeken R: Clathrindependent endocytosis. Biochem J 377: 1-16, 2004

5. Bradley SV, Holland EC, Liu GY, Thomas D, Hyun TS and Ross TS: Huntingtin interacting protein 1 is a novel brain tumor marker that associates with epidermal growth factor receptor. Cancer Res 67: 3609-3615, 2007.

6. Rao DS, Hyun TS, Kumar PD, Mizukami IF, Rubin MA, Lucas PC, Sanda MG and Ross TS: Huntingtin-interacting protein 1 is overexpressed in prostate and colon cancer and is critical for cellular survival. J Clin Invest 110: 351-360, 2002.

7. Rao DS, Bradley SV, Kumar PD, Hyun TS, Saint-Dic D, Oravecz-Wilson K, Kleer CG and Ross TS: Altered receptor trafficking in Huntingtin Interacting Protein 1-transformed cells. Cancer Cell 3: 471-482, 2003.

8. Bache KG, Slagsvold T and Stenmark H: Defective downregulation of receptor tyrosine kinases in cancer. EMBO J 23: 2707-2712, 2004.

9. Rosell R: Cancer and alterations in the endocytic pathway. Future Oncol 3: 487-489, 2007.

10. Guo HB, Johnson H, Randolph M,Lee I and Pierce M: Knockdown of GnT-Va expression inhibits ligand-induced downregulation of the epidermal growth factor receptor and intracellular signaling by inhibiting receptor endocytosis. Glycobiology 19: 547-559, 2009.

11. Mutch LJ, Howden JD, Jenner EP, Poulter NS and Rappoport JZ: Polarised clathrin-mediated endocytosis of EGFR during chemotactic invasion. Traffic 15: 648-664, 2014. 